\title{
Effect of Static Stretching On Vertical Jump Performance on Apparently Healthy Subjects
}

\author{
Balaji Palaniappan * V. Pasupatham** Deivendran Kalirathinam*** \\ * Reader in Physiotherapy, RMMC\&H, Annamalai University, Annamalai Nagar 608002, Tamil Nadu, India \\ ** Lecturer in Physiotherapy, RMMC\&H, Annamalai University, Annamalai Nagar 608002, Tamil Nadu, India \\ ***Associate professor, MAEER'S College of physiotherapy, Talegaon Dabhade,410507Pune, Maharastra \\ India
}

\begin{abstract}
With an idea to determine the effectiveness of static stretching on vertical jump performance on apparently healthy subjects, hundred male subjects on the age of 18 to 25 years in the Division of PM\&R, RMMC\&H, Annamalai University, Annamalainagar were selected for this study. Only the subjects with normal body mass index values were included. The subjects were assessed for their vertical jump performance by vertical jump test. Then static stretching was given for quadriceps, hamstrings, and calf muscles of both limbs. Immediately participants were assessed for their post stretching vertical jump performances. The values of prestretching and post stretching vertical jump performances were statistically analyzed. There was a statistical significance between pre and post values of passive static stretching and vertical jump performance. There was an increased vertical jump performance when passive static stretching applied. It may be concluded that passive static stretching can be beneficial to improve vertical jump performance while performing explosive type activities.
\end{abstract}

Key words - body mass index, vertical jump performance

\section{Introduction}

Is stretching beneficial to athletes when performing explosive type activities? This question has become a hot debate topic among exercise professional over the past years ${ }^{13}$.

The vertical jump is an explosive activity which is required by number of athletes to perform in different sports. Athletes are always striving to gain any edge they can against their competition. In spots such as basketball, soccer and volley ball, an increased vertical jump can possibly give these athletes an extra advantage; they are always trying to achieve $\mathrm{e}^{13}$.

The measurements are used primarily in athletic circles both to measure performance and as something athletes brag about themselves. The most common sports in which one's vertical jump is measured are track and field, basketball, football and volleyball, but many sports measure their player's vertical jumping ability during physical examinations ${ }^{15}$. Some professionals believe that stretching does not help to increase vertical jump performance and is just a result of proper form during execution of the jump ${ }^{14}$.

Bazett jones DM concurred that static stretching has no positive or negative impact on athletic performance ${ }^{2}$. It is not clear from prior research what the effects of stretching have on an athlete's vertical jump performance (knudson et al, 2000) ${ }^{9}$. In this study the effects of static stretching on vertical jump performance in normal subjects was analysed.

\section{Methodology}

Participants were students who were from Annamalai University, Annamalai nagar, hundred male healthy subjects were selected randomly with age group 18-25 and with normal body mass index values (18.525). Those subjects with previous lower extremity injuries and bone or joint disorders were excluded from this study.

\section{Materials}

Measuring tape, chalk for marking on the wall, weighing machine, score sheets.

\section{Procedure}

The subjects were selected according to the inclusion criteria. The subjects were assessed for their vertical jump performance by vertical jump was recorded for three trials. Then passive static stretching was given to quadriceps, hamstrings and calf muscles of both limbs. Stretching protocol consisted of 2 sets, with each stretch held for 15 seconds rest. Immediately, participants were made to perform 3 trials of vertical jumps and the values were recorded. The measurements of pre stretching and post stretching vertical jump was statistically analyzed by using paired samples test. 


\section{Stretching techniques}

\section{i) Hamstring stretching ${ }^{5}$}

Patient position: supine lying.

Hand placement and procedure:

With the patient's knee fully extended, the patient's lower leg was supported with arm or shoulder.

The opposite extremity along another aspect of thigh was stabilized with the hand or with the assistance from another person.

With the knee at 0 degrees extension, the hip in neutral rotation, the hip was flexed as for as possible.

\section{ii) Quadriceps stretching ${ }^{5}$}

Patient position; prone lying

Hand placement and procedure

The pelvis was stabilized by applying a downward pressure across the buttocks.

The anterior aspect of the distal tibia was grasped and patient's knee was flexed.

\section{iii) Calf muscles stretching ${ }^{5}$}

Patient position; supine lying

Hand placement and procedure

The patient's heel was grasped with one hand, maintaining the sub-talar joint in a neutral position, and forearm was placed along the plantar surface of the foot.

The anterior aspect of the tibia was stabilized with other hand.

The talocrural joint of the ankle was dorsi flexed by pulling the calcaneus in an inferior direction with thumb and fingers while gently applying pressure in a superior direction just proximal to the heads of the metatarsals with forearm.

\section{Vertical jump test (sergent jump) ${ }^{15}$ \\ Procedure}

The subject was made to stand side on to a wall and to reach up with the hand closest to the wall. With the feet flat on the ground, the point on the fingertips was marked. This is called as the standing reach. The subject was then made to stand away from the wall and go jump vertically as high as possible using both arms and legs to assist in projecting the body upwards, with an attempt to touch the wall at the highest point of the jump. The difference in distance between the standing reach height and the jump height was recorded as score. The three trials of jump performance were recorded.

\section{Statistical analysis and results}

The data collected were statistically analyzed for the observations namely before and after stretching by using paired ' $t$ ' test for the equality of the means of those two observations.

Table1

Mean and standard deviation for pre-stretching and post-stretching vertical jump performance

\begin{tabular}{|l|l|l|l|}
\hline & $\mathrm{N}$ & Mean & SD \\
\hline Pre- test & 100 & 39.5860 & 7.48973 \\
\hline Post- test & 100 & 43.9932 & 7.09866 \\
\hline
\end{tabular}

The mean pre stretching vertical jump performance and the standard deviation values were found to be 39.5860 and 7.48973. Likewise the mean post stretching vertical jump performance and the standard deviation were found to be 43.9932 and 7.09866 .

\section{Paired' $t$ ' test}

Table 2

\begin{tabular}{|l|l|}
\hline $\mathbf{t}$ & 13.446 \\
\hline Sig. & $\mathrm{P}=.000$ \\
\hline
\end{tabular}

The mean jump performance was compared by using paired ' $t$ ' test and the $t$ and $p$ values obtained were 13.446 and 0.00 respectively, which is significant.

Therefore it implies that there exists a significant difference in pre and post stretching vertical jump measures. 


\section{Discussion}

The results of this study were shown to be statistically significant. There was an increased vertical jump performance when passive static stretching was applied, thereby suggesting that static stretching can be beneficial to improve vertical jump performances while performing explosive type activities such as basket ball, soccer and volley ball etc.

The result of this study was similar to the study conducted by Tom Evans ${ }^{14}$, where there was beneficial effect of static stretching on vertical jump performance and contradictory to the study conducted by young WB $^{18}$, where static stretching had a negative influence on vertical jump performance. For this study, 100 male normal subjects were selected according to the inclusion criteria. The subjects were assessed for their initial vertical jump performances by vertical jump test as suggested by Augsustsson $\mathbf{J}^{1}$. Then passive static stretching was applied to quadriceps, hamstrings and plantar flexors as two sets, with each stretch held for 15 seconds rest as suggested by Robbins $\mathbf{J W}^{11}$. Post stretching vertical jump performance was assessed immediately for each and every participant.

Thomas Kurz suggested that static stretches were not beneficial in warm-up as the isometric tensions will tire and decrease the coordination of sportsman ${ }^{13}$. Depino GM held that effect of static stretching lasted only three minutes after cessation of the stretching protocol ${ }^{7}$. The study conducted by spernoga SG concurred that modified hold relax stretches produced significantly increased hamstring flexibility that lasted 6 minutes after the stretching protocol ended ${ }^{12}$.

De Weijer, in this, concluded that hamstring length can be maintained for up to 24 hours using static stretching ${ }^{8}$. There are controversies in the duration of effectiveness of static stretching due to different results of various studies.

In this study, post stretching vertical jump measures was assessed immediately after stretching for apparently normal and healthy subjects. This study can be further enhanced by assessing the intervals and investigating the association between stretching and practice jumps on explosive force production and vertical jump performance for athletes.

\section{Conclusion}

It was concluded that there was significant improvement in vertical jump performance in apparently normal and healthy male subjects after static stretching of the three groups of muscle viz., the quadriceps, hamstrings and the calf muscles. Passive static stretching can be beneficial to improve vertical jump performances while performing explosive type sports activities.

\section{References}

[1]. Augustsson J. Thomee R. "Ability of closed and open kinetic chain tests of muscular strength to assess functional performance." Scand J Med Sci sports, 2000 Jan: 10(3): 164-8.

[2]. Bazett-Jones DM - Gibson MH, Mcbride JM. "Sprint and vertical jump performances are not affected by six weeks of static hamstring stretching." J. Strength cond Res. 2008 Jan; 21(1):25-31.

[3]. Behm DG, Kiblle A. "Effects of differing intensitites of static stretching on jump performance." Eur J Appl Physiol. 2007 Nov; 101(5): 587-94.

[4]. Bradley PS. Olsen PP. "The effect of static, ballistic, and proprioceptive neuromuscular facilitation stretching on vertical jump performance." J Strength cond Res. 2007 Feb; 21(1) : 223-6

[5]. Carolyn Kisner - Lynn Allen Colby, Therapeutic Exercise -Foundation and Techniques 4th edition.

[6]. Church JB - Wiggins MS, Moode FM, Crist R. "Effect of warm-up and flexibility treatments on vertical jump performance." $J$. Strength Cond Res. 2001 Aug; 15(3): 332-6.

[7]. Depino GM, Webright WG, Arnol BL. "Duration of Maintained Hamstring Flexibility After Cessation of an Acute Static Stretching Protocol." J Athl Train 2000 Jan: 35 (1): 56-59.

[8]. De Weijer VC, Gorniak GC, Shamus E. "The effect of static stretch and warm-up exercise on hamstring length over the course of 24 hours." J orthop sports Phys Ther. 2003 Dec; 33(12): 727-33.

[9]. Knudson D, Bennett K, Corn R, Leick D. Smith C. "Acute effects of stretching are not evident in the kinematics of the vertical jump." J. Strength cond Res 2001Feb; 15(1): 98-101.

[10]. Power K, Behm D-Cuhill F. "An acute bout of static stretching: effects on force and jumping performance." Med Sci. sports exerc. 2004 Aug; 36 (8): 1839-96.

[11]. Robbins JW. Scheverman BW. "Varying amounts of acute static stretching and its effect on vertical jump performance." $J$ Strength cond Res 2008 May; 22(3):781-6.

[12]. Spernoga SG, Uhl TL, Arnold BL, Gansneder BM. "Duration of Maintained Hamstring Flexibility After a One-time, Modified HoldRelax Stretching Protocol." J Atthl Train 2001 Mar: 36(1) : 44-48.

[13]. Thomas Kurz. "Stretch yourself - Stretching and injuries."

[14]. Tom Evans, "The effects of static stretching on vertical jump performance." Trie graduate college of Marshall University. May 2006. Vertical jump test- wikipedia.

[15]. Wallmann HW, Mercer JA. "Surface electromyographic assessment of the effect of static stretching of the gastrocnemius on vert ical jump performance." J Strength CondRes. 2005 Aug: 19(3): 684-8.

[16]. Woolstenhulme MR, Griffiths CM. "Ballistic stretching increases flexibility and acute vertical jump height when combined with basketball activity. J Strength Cond Res 2006 Nov; 20(4): 799-833.

[17]. Young W. Elias G. "Effects of static stretching volume and intensity on plantar flexor explosive force production and range of motion." J sports med phy fitness. 2006. Sep: 46(3): 403-11. 